\title{
A Roadmap to Nextgen Aircraft Control for Last Mile Delivery Drones
}

\author{
Cesar A. C. Marcondes, Denis S. Loubach, Elton F. Sbruzzi, Filipe A. N. Verri, Johnny C. Marques, Lourenço A. \\ Pereira Jr, Marcos R. O. A. Maximo, Vitor V. Curtis
}

\begin{abstract}
According to recent studies, unmanned aerial vehicles (UAV) can play a game-changing part in terms of cost reduction and speed increase to address the last-mile delivery (LMD) problem and also to attend emergencies. Last-mile delivery services are getting more and more relevant, especially when in times where social distance is required. Given this scenario, our paper introduces a cyber-physical (CPS) system roadmap propose applicable for last-mile delivery drones. The proposed CPS guidelines are based on the concept of system of systems to enable an emerging behavior towards smart cities' governance. In this paper, we also discuss topics from air space control and reservation throughout communication infrastructure and decentralized control supported on a blockchain.
\end{abstract}

Index Terms-Unmanned aerial vehicles (UAV), Drones, Lastmile delivery (LMD), Cyber-physical systems (CPS), Communication, Tradable permit model (TPM), Blockchain.

\section{INTRODUCTION}

$\mathbf{U}$ NMANNED aerial vehicles (UAV) can play a gamechanging part in terms of cost reduction and speed increase to address the last-mile delivery (LMD) problem and also to attend emergencies [1].

The last-mile delivery services are getting more and more important, especially when in times where social distance is required [2]. Studies indicate that the last-mile is one of the most expensive, inefficient, and polluting parts within the supply chain. It can reach from 13 to $75 \%$ of the total supply chain cost in given scenarios [3]. Also, last-mile delivery services are a concern for the major e-commerce retailers including Amazon, Walmart, and Alibaba. In this context, UAVs, also known as drones, are of special interest [4]. However, other LMD solutions point out that complex traffic environments can still be explored with autonomous terrestrial vehicles [5].

According to [6], the usage of drones for delivery purposes can have at least four main advantages: (i) autonomy, (ii) avoidance of traditional road network, (iii) cost, and (iv) speed. Despite these advantages, there are lots of open issues, such as airspace utilization, payload capacity planning, auto-pilot, and navigation in shadow areas.

Drones can be remote-controlled or even fully autonomous, depending on regulations. These regulatory agencies' policies vary from place to place. Generally, drones are classified into high altitude platforms (HAP), e.g. 17+ km, or low-altitude platforms (LAP), e.g. tens of meters to few kilometers. HAPs are mostly regarded as quasi-stationary and present better

Authors are with the Computer Science Division, Aeronautics Institute of Technology - ITA, São José dos Campos, SP, 12228-900 Brazil endurance to face a few days to months campaign. On the other hand, LAPs are more agile, cost-effective, and can be recharged in a much faster way.

Drones are also categorized depending on their aeronautical frame: they can be fixed- or rotary-wings in this sense. The former, such as small planes, have higher speeds and can carry more load, but they need to keep flying forward with relatively high speeds to stay in the air, making it harder to perform sharp maneuvers. The latter can be represented by a quadrotor drone that can hover at lower speeds or even in place, however, their flight autonomy is limited to less than one-hour [7] given nowadays electronic batteries technology. A hybrid drone is also possible, i.e. having fixed- and rotary-wings on the same platform.

As mentioned by Alwateer and Loke [8], drones are on the edge of the delivery service. This can be confirmed as initiatives in the air traffic management (ATM) system, including SESAR and NextGen, indicate ongoing development along with future communication infrastructure preparations.

Aerial delivery may impact merchandise, courier, food delivery, humanitarian aid, and passenger transport [9]. The last is considered very ambitious but it is already planned and being scratched. These applications require agents (e.g. UAV's or drones) to have a plan and execute delivery routes taking into account cost and time minimization while avoiding collisions with other agents and the environment.

Given this overall picture, our paper introduces a cyberphysical (CPS) system's roadmap propose applicable for lastmile delivery drones. The proposed CPS guidelines are based on the system of systems' concept to enable an emerging behavior towards smart cities' governance. Here, we discuss topics from air space control and reservation throughout communication infrastructure and decentralized control supported on a blockchain.

In Section II, we introduce our proposed cyber-physical system for last-mile delivery. In the following sections, we detail each aspect of the system: airspace reservation and mobility model (Section III), communications (Section IV), navigation and path planning (Section V), safety and certification (Section VI), and decentralized control (Section VII). Finally, Section VIII provides final remarks and research directions.

\section{Proposed Cyber-Physical System For LMDD}

The cyber-physical system for last-mile delivery drone (LMDD) comprises airspace, drones and navigation, communication infrastructure, geofencing service, safety points, and 
end-points. Together they form a system of systems (SoS) and must be tightly coupled to produce an emerging behavior to enable smart city governance. We discuss each of these subsystems next.

a) The airspace control and reservation: A groundbreaking feature is to bring the airspace's reservation and utilization to a civilian context, allowing the private sector to operate without the burden of the human-in-the-loop process. As a smart city resource, the airspace can be segmented and objected to allocating a specific space to a tenant. We propose an economic model to permit a sustainable with revenue and constant upgrading, conducive to a public-private partnership.

b) Geo-fencing service: The airspace segmentation allows reserving a specific location for a given aircraft, defining four-dimensional fencing block: altitude, longitude, latitude, and time. The block size is location dependent, but small sizes supply flexibility and efficiency to airspace utilization, granted after an auction process. The complete workflow consists of a sequence of bids where players (organizations willing to operate in the system) intent to acquire a set of fencing blocks to form a path. In the end, a blockchain system persists all exchanges. Then, the player submit a mission to start operation. It results in an auction-oriented mission, where only authorized players can operate in a path.

c) Drones and navigation: Drones need to be equipped with sensors and communication radios to provide safety and reduce variance in operation. A player-owned ground control station (GCS) must manage its fleet, enabling adaptive algorithms to tackle deviances dynamically. Furthermore, the smart city administration can interrupt ongoing missions, which is crucial to implement city-wide safety services concerning weather conditions, such as wind, rain, fog, and snow.

d) Communication infrastructure: The communication infrastructure must perform missions accordingly. It requires massive machine type communications (mMTC) and ultrareliable low-latency communications (URLLC). It is also important to incorporate security aspects for authentication and authorization.

e) Safety points: A set of physical points must be available throughout the airspace, serving as a safe spot in which drones can land in case of unappropriated operation conditions. Due to the operational environment's natural volatility, a safe point is strategical to emergencies including battery drained, erratic weather, among others.

f) End-points: They correspond to places where missions start and also where they end. The starting terminal is potentially a logistic center where operators dispatch packets to delivery, yet they realize advanced approaches such as Amazon's zeppelin. At the delivery place (the mission endpoint) must exist a spot to automatically receive the packet. It can be static (in buildings or houses), or mobile like the top of cars (Uber use case).

\section{Airspace Reservation AND Mobility Model}

The volume of urban UAV's is expected to grow in the next years. One of the foreseen challenges of this growth is how to manage that traffic. In this matter, we argue that schemes available in the literature about urban motorways traffic (UMT) can be adapted to urban UAV traffic (UAT).

Even though many traffic models have been proposed and employed for UMT [10], their application to UAT is not trivial. We highlight the following reasons:

- Constraints related to the airways are inherently less restrictive than motorways. As a result, optimal flight planning in airways commonly becomes unfeasible for many agents, e.g. drones. Constraints in the airspace must be imposed carefully to achieve a reasonable scenario; and

- Although airspace congestion is less likely than motorways congestion, the consequences regarding failures are far more serious. Thus, safety considerations must not be undermined.

Traditional airspace traffic management schemes avoid congestions, and collisions altogether, by using a central unit. Such a unit plans strict paths for each aircraft and oversees them. However, traditional schemes are not appropriate to deal with the responsiveness requirements of LMD.

Tradable permit model (TPM) is a novel approach to cope with capacity allocation that uses a market mechanism to assign rights to users of a particular resource [10]. Permit schemes have received growing attention in the academic literature. Permit's decentralized nature brings advantages over centralized approaches [11]. In the context of LMDD, the airspace is the resource of interest.

A number of researchers study the coordination of swarms of cooperating drones [12] or the optimization of planned routes globally [13]. We argue that both approaches are not suitable for managing the mobility of LMDD if it is taken individually. Let's consider the players, which are companies or individuals that own LMDDs and desire to use the airspace. First, in real-world scenarios, it is unfeasible to force different players to use the same given strategy to coordinate their drones. Moreover, a central entity that calculates the optimal route for each LMDD in the airspace would require an impractical computational power.

In view of this, we propose a TPM that serves as a guideline to nextgen aircraft control for LMDD. Related schemes have been proposed [11], [12], [13], [14]. However, our approach raises the novel challenge of cooperation and competition in a much more realistic scenario. We describe such a mobility model in the following subsections.

\section{A. Free-Market Permit Concession}

In real scenarios, many players have specific interests in the usage of the airspace. Some interests, however, will certainly conflict. For instance, two players might want to use the same space at the same time to accomplish the delivery mission within the expected time.

Solving the conflicts in a decentralized cooperative way, e.g. relying on common strategies among different players, would require impractical regulatory and expensive oversight mechanisms. Conversely, in the competitive view, each player must obtain beforehand a permit to use the desired airspace at the desired time. In this context, a permit is an authorization to 
use a specific volume in the airspace for a specific timespan. As a result, the regulatory oversight would focus on inspecting whether LMDDs have a permit instead of scrutinizing each flight for compliance to a given policy.

Still, a centralized permit concession procedure has some limitations: a) optimizing routes, airspace usage, and financial gains becomes computationally unfeasible as the number of players increases; b) response time of the central system might not be feasible for LMDD; c) a centralized system is not robust to failure or attacks, making it as a single point of attack; and d) the downtime of the system would result in catastrophic, i.e. financial-related, outcomes.

As a possible and strong solution, the free-market distributed permit concession is in place. In this scheme, each player would bid for a sequence of adjacent permits that accomplishes its mission. Once a player acquires permits, it can freely use or trade them. Each permit is always owned by some entity. That means regions of the airspace, and consequently, a portion of the permits would be previously assigned to public- or private-sector organizations.

To assert the feasibility of such an approach, we designed a multi-agent system simulation of the TPM scheme for UAV. In [15], we simulate players in a tradable permit model under different arrival rates, i.e. number of players competing in the auction, by focusing on two main perspectives: a) an assessment on the time and cost agents have to complete their mission; and b) an estimate of the effective airspace usage. The results from that research showed that even a naïve decentralized competitive approach yields satisfactory results even under high traffic conditions. Also, there were evidences that smarter agents can behave even better. From a practical perspective, such a study found out interesting properties emerging from the agents' collective behavior that could drive how airspace policies are defined.

In our proposal, some research questions regarding the parameters of the concession mechanism are central. For instance, one must address the kind of auction and the appropriate time/space scales of the permits for each practical scenario.

\section{B. Optimal Usage of the Airspace and Collision Avoidance}

Although we argue that full cooperative schemes and full centralized schemes are not practical, central cooperative instructions are unavoidable inside the player's perspective.

Once a player owns the sequence of permits to use the airspace, it uses the respective airspace as desired, of course, by respecting the security and safety regulations. For instance, each player surely owns several LMDDs to fulfill many delivery missions. Then, it needs to coordinate its resources to optimize its gains while satisfying the space and time constraints.

Given a set of drones owned by the same player, they can work cooperatively. We discuss the details about the coordination of drones, including collision avoidance, at this level in Section V. Another fundamental aspect of coordination is the communication infrastructure and the requirements of the real-time control system.

\section{COMmunicAtion System InFRASTRUCTURE}

To cope with the last-mile delivery drone system's complexity, we assume full connected infrastructure and battery-bound operation requirements. In this communication system infrastructure context, researches also take into account common UAV communication protocols over cellular links [16], passive sensor nodes in a local sensor network [17], and mobile target tracking [18].

We consider the smart city scenario typical for deployment and key-technologies to support this system's viable implementation. Thus, it will be feasible to exist communication amongst the aircraft in the system and aircraft to the ground base station, resembling vehicle to vehicle (V2V) and vehicle to infrastructure (V2I) communication model. Offload of processing must be another resource available as a service in this infrastructure, possibly in the form of multi-access edge computing (MEC).

Our tradable permit model for last-mile delivery [15] enables large-scale operations with multiple layers in altitude, each one segmented to form four-dimensional geo-fencing units. Hence, many massive machine type communications problems can arise, as drones share the same media for communication. On the other hand, the ground control station requires an ultra-reliable low-latency communication channel to provide offload of challenging computational tasks involved in achieving global consensus of operations. Each drone sends the sensor's information to GCS periodically, allowing the creation of discrete-time snapshots, optimizing configuration parameters, and sending the new setup back to the aircraft. Communications play a critical role in this feedback control loop, acting as a bottleneck in this infrastructure.

Aircraft to aircraft (A2A) communication is typically in line of sight and makes it possible to implement essential services such as collision avoidance and trajectory planning. A2A impacts not only in the same origin fleet but also on other tenants using the airspace. Problems here include spectral sharing and security issues involving authentication and authorization. In this context, there is some protocols including Mavlink. It is a protocol for message passing with drones, and so security needs severe scrutiny. Aircraft to infrastructure (A2I) communication, as a combination of access-network and computational resources, enables the offload of critical functions to the edge. It includes a global vision of a tenant fleet, and the smart city takes control of the whole system to provide safety (emergency or catastrophic situations). 5G and beyond have addressed the problems we list here. However, it requires more workload characterization to expose new knobs not yet pondered for A2I.

Those requirements can culminate in new studies to provide realist service-level agreements (SLA) and quality of service (QoS) to LMDD scenarios. The presence of multi-access edge computing (MEC) is essential to cope with communications and processing demands. Latency is another curcial metric, and all the elements in the communication system, including front-haul and backhaul, must carefully be engineered to meet SLA and QoS constraints. The GCS efficiency is as good as the capacity of the system in providing a lower latency experience. 
Public-private partnerships can be meaningful for LMDD to deploy adequate infrastructure to operations. In this sense, our economic model of trade permit model supports a sustainable modality, providing CapEx and OpEx financial resources.

Battery-aware aircraft provides efficiency to operations as it can self-adapt to condiction dynamically. During operations, expected and unexpected events impact energy consumption, causing deviance on mission-planned versus actual conditions. The main problem is in the sources of uncertainty, as on commissioning is possible to estimate the minimal capacity planning required. For example, due to uncontrollable weather changes, more processing power can arise during the mission, making it differ from the original. Thus, we can divide resource utilization into two: bare-minimal and exceeding. The former is about the basal resources consumed and known before the mission. The latter is the unexpected and unknown events that emerge dynamically. Processing the exceeding demands MEC, provided an ecosystem with enough latency, reliable, and cloud-enabled computing providers.

Such an infrastructure allows players to operate safely and the smart city to implement an unmanned aircraft system (UAS) traffic management architecture. Path planning works as a core service for both and needs in-depth analysis.

\section{Path Planning and Execution}

In a real context, UAVs can be simply considered as one more transportation vector to existing supply chain management and logistic systems. In such systems, decision-making should consider not only profitability, but also service quality, equity, consistency, simplicity, reliability, and externalities [19]. The interrelations of these enterprise-wide challenges play a key role, especially when seeking optimal solutions. For LMDD, this holistic view is even more important once it represents a $13-75 \%$ economic opportunity of the total supply chain [3].

Operations research (OR) is a import research field that has been applied as an integrating decision-making tool for various industries over the last decades, resulting in significant savings. Among the benefits to the LMDD, it includes a wellestablished research area, systems with decades of developments, easy and cheap integration with current systems, and some level of isolation during the problem modeling.

In OR, the vehicle routing problem (VRP) is the classical approach when planning optimal routes for a set of delivery agents from a depot to a set of geographically scattered customers, subject to constraints [20]. It is a rich and broad area of research with many variations that generalizes the wellknown NP-hard traveling salesman problem (TSP), so VRP may be better defined as a class of problems.

According to the taxonomies of [21], [22], our problem can be better described as a class of capacitated VRPs (CVRPs) and distance-constrained VRP (DCVRP), respectively, when the modeling has to consider limited carrying capacity for the vehicles and the total length of the path in a route cannot exceed a maximum limit. The VRP may be considered with many other variations related to the LMDD, such as: VRP with time windows constraints (VRPTW), where allowable times or time intervals are associated with every customer; termed periodic VRP (PVRP), when the scheduling extends from one to many days; and VRP with pickup and delivery (VRPPD) or VRP with Backhauls (VRPB), where customers may return items.

Many solution strategies have been devised for VRP [20]: exact algorithms often employ formal optimization methods with optimality guarantees but are limited to solving relatively small instances of the problem; classical heuristics use heuristics especially tailored to VRP; and metaheuristics employ general black-box optimization algorithms, such as simulated annealing and genetic algorithms. Despite the loss of optimality and feasibility guarantees, in practice, metaheuristics typically work well and are able to find close to optimal solutions for large instances of VRP in a reasonable amount of time [20].

One example of delivery drones as VRP is proposed by Dorling et al. [1]. They model the problem through mixedinteger linear programming (MILP), providing minimum time and minimum cost variants. Their model considers the costs related to acquiring and operating the drones, while also taking into account delivery time and budget constraints. To compute operation costs, they consider an energy expenditure model based on physical principles which are also backed by experimental data. By using the commercial solver CPLEX [23], they are able to solve the optimization problem exactly for up to eight agents. Also, they show that an alternative solver based on simulated annealing obtains close to optimal solutions to instances of up to 500 drones in less than a minute. From a practical perspective, we expect that the choice of solution strategy (exact or metaheuristic) will depend on the number of drones a particular player has.

Although the integration of these challenges is crucial for better solutions, optimizing all of them at once may become infeasible and unrealistic, e.g. some decisions must be taken only after the realization of an event. In order to handle the complexity of the LMDD, our solution [15] proposes a hierarchical strategy as more promising. The decision-making can be described from the player perspective and its dynamics with the system and other players with three phases:

- Cooperative: cooperation of its resources to address the logistic optimization;

- Competitive: competition in the market with other players for permits; and

- Navigation: response to unexpected and rare events.

The cooperation of resources is the traditional VRP and can be solved by the before-mentioned solutions. The competition in the market imposes unknowns to the optimization problem that are commonly described as stochastic variables. Actually, LMDD may impose many other stochastic behaviors, e.g. weather conditions, battery autonomy, and others.

There are many ways to integrate the competition in the VRP, however, the most direct approach is by modeling the cost of the permits as part of the distances to be traversed by a UAV. Verri et al. [15] shows that players can obtain routes close to the optimal length and cost even in regions with very dense competition. In other words, we expect the players can focus only on the neighboring permits it desires, 
ignoring most of the other permits. We also expect prices close to homogeneous in regions with low competition.

A common approach for such complex problems is to implement a two or more stage model. These models are especially interesting when just part of the variables must be defined before some realization, while the other decisions may be benefited from the information of the realizations. After the events, a re-optimization is required to adapt the decision to a new reality [24]. In this case, the recourse decision must be feasible or there is no way to optimize it. Another advantage of this approach is that the original problem is broken into smaller problems, making the solution more practical.

For the LMDD, the natural stages would be first to define the desired routes based on the demand of the permits and, after the realization of the auction, re-optimize the decisions based on the holding permits. This adaptation may also be performed continuously in a time-window or event realization approach, i.e. re-optimization for part of the problem can be performed at each fixed period or after some important event. Of course, the time-window will depend on many factors such as: computing resources, size of the problem, kind of events, competition of permits. However, we expect at least a daily frequency for planning routes of UAVs in a dense region.

Depending on the nature of the event, online (or realtime) decisions will be required for safety or security reasons. For safety purposes, robust behaviors to stochastic variables may be required by the authorities in order to avoid harmful outcomes. Players may also benefit from it in order to avoid unexpected events with high costs.

The most difficult challenge imposed by UAVs is the command and control (CC) of the LMDD airspace. It requires an infrastructure with real-time monitoring and responses to perform fast maneuvers to avoid collisions. This is important for path planning and navigation once it can increase the cost of the routes and reduce battery autonomy during unexpected events.

The impact of this solution is relevant once it avoids the need for various re-optimizations considering that the collision detection system will be activated during the navigation just in rare cases.

Inter-agent collision avoidance is typically not dealt with in VRP. This is theoretically possible and the interested researcher could in principle benefit from the literature regarding multi-agent trajectory planning through model predictive control (MPC) [25], [26], where inter-agent collision avoidance is achieved by imposing additional constraints in an optimization problem. MPC-based approaches for trajectory planning are also based on MILP. Nevertheless, considering current processing power and optimization solver technology, we consider that direct inclusion of inter-agent collision avoidance in VRP would make the optimization problem computationally intractable, and certainly not suitable for real-time applications such as the one proposed here.

In this case, an inner layer would coordinate the UAVs to execute the planned routes while dealing with inter-agent collision avoidance. Since drones are relatively small with respect to the air space, and collision avoidance would be rare, we do not expect frequent conflicts, so the hierarchical division should not result in considerable loss of optimality.

There is a vast literature in robotics path planning that may be employed [27], [28]. Due to the existence of a central base that may communicate with the drones through a communication infrastructure, sophisticated control algorithms such as MPC-based trajectory planners may be used [25], depending on the number of UAVs and the available computational resources. These algorithms could operate in a closed-loop fashion, taking into account recent information collected by the drones and the communication infrastructure. Independent of the approach taken, a low-level local obstacle avoidance based on the drone's sensor readings and running locally on the drone should always be present to permit quick reactions to unexpected obstacles and continue mission execution when communication with the base is lost.

Finally, a large delivery may exceed the carrying capacity of a single drone. In this case, the delivery may be carried out by multiple drones and techniques for UAV formation control may be employed.

\section{Regulatory DiRections}

The use of drones, from recreational flying to commercial uses, is a concern for safety and certification. Whether manned or unmanned aircraft, the Federal Aviation Administration (FAA) requires that all operators follow specific guidelines for the operations they request. Currently, the Part 135 addresses a set of rules for the certification of UAV. Basically, the Part 135 is not dedicated to UAV, but is the only path for small drones to carry the property of another for compensation beyond visual line of sight.

UAV consider that operation is outside aircraft, and no human is onboard, but vehicle is controlled from the ground. The UAV certification has some regulation for vehicles and operators, to ensure safety. The use of drones, in connection with a business activity, is allowed in many countries, but under very strict conditions. In most countries one will need different authorizations or licenses from the National Aviation Authority before starting any operation.

However, for autonomous UAV, no regulations are available at this point, and is very unclear if current regulation for manned aircraft vehicles will fit this need. The operator is an automatic on-board pilot system (AOBPS), which refers to the level of automation of the drone when, at highest level of automation, this is about piloting functions and on-board decision making with little or no human intervention. In this paper, we are considering the AUAV are drones used for package delivery.

We should specially analyze the certification regulations in the following areas:

- Safety assessment; and

- Software and hardware certification.

\section{A. Safety Assessment}

This process is focused on identifying functional failure conditions leading to hazards that are closely related to development assurance levels (DALs). Currently, there are five DALs, as presented in Table I. 
Table I: Hazard Classification and DAL

\begin{tabular}{lll}
\hline $\begin{array}{l}\text { Hazard } \\
\text { Classification }\end{array}$ & $\begin{array}{l}\text { Development } \\
\text { Assurance } \\
\text { Level }\end{array}$ & $\begin{array}{l}\text { Maximum } \\
\text { Probability per } \\
\text { Flight Hour } \\
\text { (MPFH) }\end{array}$ \\
\hline Catastrophic & $\mathrm{A}$ & $10^{-9}$ \\
Hazardous & $\mathrm{B}$ & $10^{-7}$ \\
Major & $\mathrm{C}$ & $10^{-5}$ \\
Minor & $\mathrm{D}$ & $10^{-3}$ \\
No Effect & $\mathrm{E}$ & - \\
\hline
\end{tabular}

So some questions are needed to be answered. Is the MPFH of $10^{-9}$ acceptable for the most critical hazard classification of system failures for AUAV, specially for LMDD vehicles? We believe that yes, this is acceptable. Basically, if a aircraft with about 600 passengers uses this concept, why a small drone for LMDD can't uses the same approach? Another question is the industry of drones perform a traditional safety-assessment process during drone project, according to our research and literature review: no, they do not. Then, regulations can be used as is, but industry should adapt their development processes to allow compliance with regulations.

\section{B. Software and Hardware Certifications}

The RTCA DO-178C and DO-254 has been used as an acceptable mean of compliance for software and hardware to ensure safety in the appropriate level of rigor, according to the safety assessment. However, the AOBPS will coordinate the operation of other systems of the AUAV. So, the study of additional characteristics must be performed to ensure if the software and hardware level must be increased to level $\mathrm{A}+$, as traditionally, software and hardware systems follow a process rigor considering non-autonomous flight. Additionally, currently software standards, as DO-178C, use a concept that behavior must be deterministic and the software is specified with an enormous amount of detailed requirements that describe its behavior. The use of technologies such as machine learning, which make the solutions present an evolutionary behavior, cause some incompatibility with the way these regulations were conceived.

\section{A DeCEntralized Control Supported By BLOCKCHAIN}

As a cyber-physical system representing a critical infrastructure and composed of multiple players, the system's fundamental responsibility concerns how data dissemination throughout the communications systems (potentially the Internet) occurs. Cybersecurity is the main focus of this service, guaranteeing integrity, availability, consistency, resistance to distributed denial of service (DDoS) and 51\% attacks, and authorization and authentication. Blockchain-based systems provide a robust framework to this end, allowing rapid evaluation of different alternatives and configurations to scenarios in future study cases.
The airspace reservation procedure consists of a sequence of bids to acquire a 4-dimensional (latitude, longitude, altitude, and time) slot, which we call permit. Players race to achieve a list of adjacent permits, and, during a period, the reservation converges to a consistent state, where each player has the right to use a path. Thus, a set of transactions can be stored to a public resource, as players use the airspace concurrently, whereas the entities responsible for the air traffic management govern the system utilization. The sharing mechanism is a critical point of failure in this context: malicious users can intentionally manipulate data to induce error-prone operational conditions, potentially causing malfunctioning leading to hazard situations.

Adopting a blockchain-based system for data dissemination leverages benefits to the system as a whole. When a new set of transactions is ready, it is signed and included as a block after being validated by more the half of peers presented in the system. The system hashes all data and stores it in a Merkle tree structure. Tampering the information in blocks will require substantial computing resources, as players and other entities store replicas. It is worth mentioning that the game-theoretic method to insert information must be under investigation. Consensus models, such as proof-of-work (POW), can lead to advantages to specific scenarios. However, other schemes can outperform it, e.g. proof-of-event, proof-of-stake, and proofof-authority.

The workload imposed on the system is dependent on the permits' size. More oversized permits decrease the number of combinations to form a path, and vice-versa. Thus, smaller permits occasion on more degrees of freedom, which increases the number of transactions in the system. Consensus mechanisms dominate the throughput and consequently impact availability. A careful study investigating different mechanisms is encouraged and can yield useful insights to tackle the tradeoff of the variables consensus speed and security level.

The replicas' distributed nature in the blockchain allows consistency, serving as a fault-tolerance feature and a source of truth in the system. Another advantage of it is the robustness in the face of DDoS attacks since there is no centralized entity in the system. A malicious user intending to insert poisoned data need to compromise $51 \%$ of the participants in the system. Assuming blockchain is flexible enough to scale-out the nodes participating in the process, $51 \%$ attacks are costly, and there are mechanisms to mitigate them, e.g. adding more nodes.

\section{SUMMARY}

In this paper, we tackled an emerging topic for research: last-mile drone delivery. Particularly, we proposed a cyberphisycal system to this topic in order to enhance the smart city governance. Our system is a system of systems (SoS) which is composed by six different subsystems: (1) airspace, (2) drones and navigation, (3) communication infrastructure, (4) geofencing service, (5) safety points and (6) end points. These subsystems is expected to work together in order to increase the quality of the proposed cyber system.

For the future works, we intend to work into two different directions. The first direction is the micro level research. 
The development of each one of the six subsystems of our cybersystem individually. And the second direction is the macro level research. The integration of those subsystems aiming to enhance our cybersystem as a whole.

Our paper tackled an emerging topic: last-mile delivery drones. Here, we propose a cyber-physical system's applicable for last-mile delivery drones, as a series of guidelines. The

\section{REFERENCES}

[1] K. Dorling, J. Heinrichs, G. G. Messier, and S. Magierowski, "Vehicle routing problems for drone delivery," IEEE Transactions on Systems, Man, and Cybernetics: Systems, vol. 47, no. 1, pp. 70-85, 2017.

[2] V. Chamola, V. Hassija, V. Gupta, and M. Guizani, "A comprehensive review of the covid-19 pandemic and the role of iot, drones, ai, blockchain, and $5 \mathrm{~g}$ in managing its impact," IEEE Access, vol. 8, pp. 90 225-90 265, 2020.

[3] J. Olsson, D. Hellström, and H. Pålsson, "Framework of last mile logistics research: A systematic review of the literature," Sustainability, vol. 11 , no. $24,2019$.

[4] D. Schneider, "The delivery drones are coming," IEEE Spectrum, vol. 57, no. 1 , pp. 28-29, 2020.

[5] B. Li, S. Liu, J. Tang, J. Gaudiot, L. Zhang, and Q. Kong, "Autonomous Last-Mile Delivery Vehicles in Complex Traffic Environments," Computer, vol. 53, no. 11, pp. 26-35, nov 2020.

[6] Q. M. Ha, Y. Deville, Q. D. Pham, and M. H. Hà, "On the min-cost traveling salesman problem with drone," Transportation Research Part C: Emerging Technologies, vol. 86, pp. 597 - 621, 2018. [Online]. Available: http://www.sciencedirect.com/science/article/ pii/S0968090X17303327

[7] M. Mozaffari, W. Saad, M. Bennis, Y. Nam, and M. Debbah, "A tutorial on uavs for wireless networks: Applications, challenges, and open problems," IEEE Communications Surveys Tutorials, vol. 21, no. 3, pp. 2334-2360, 2019.

[8] M. Alwateer and S. W. Loke, "On-drone decision making for service delivery: Concept and simulation," in 2019 IEEE International Conference on Pervasive Computing and Communications Workshops (PerCom Workshops), 2019, pp. 937-942.

[9] E. Frachtenberg, "Practical drone delivery," Computer, vol. 52, no. 12, pp. 53-57, 2019.

[10] W. Fan and X. Jiang, "Tradable mobility permits in roadway capacity allocation: Review and appraisal," Transport Policy, vol. 30, pp. 132-142, nov 2013. [Online]. Available: https://linkinghub.elsevier. com/retrieve/pii/S0967070X13001352

[11] D. K. Brands, E. T. Verhoef, J. Knockaert, and P. R. Koster, "Tradable permits to manage urban mobility: Market design and experimental implementation," Transportation Research Part A: Policy and Practice, vol. 137, pp. $34-46,2020$.

[12] J. Wang, C. Jiang, Z. Han, Y. Ren, R. G. Maunder, and L. Hanzo, "Taking drones to the next level: Cooperative distributed unmannedaerial-vehicular networks for small and mini drones," IEEE Vehicular Technology Magazine, vol. 12, no. 3, pp. 73-82, 2017.

[13] T. Akamatsu and K. Wada, "Tradable network permits: A new scheme for the most efficient use of network capacity," Transportation Research Part C: Emerging Technologies, vol. 79, pp. 178-195, 2017.

[14] Z. R. Bogdanowicz, "Flying swarm of drones over circulant digraph," IEEE Transactions on Aerospace and Electronic Systems, vol. 53, no. 6, pp. 2662-2670, 2017.

[15] F. A. N. Verri, C. A. C. Marcondes, D. S. Loubach, E. F. Sbruzzi, J. C. Marques, L. A. P. Júnior, and M. R. O. A. Máximo, "An analysis on tradable permit models for last-mile delivery drones," IEEE Access, vol. 8, pp. 186279-186290, 2020.

[16] J. Morales, G. Rodriguez, G. Huang, and D. Akopian, "Toward uav control via cellular networks: Delay profiles, delay modeling, and a case study within the 5-mile range," IEEE Transactions on Aerospace and Electronic Systems, pp. 1-1, 2020.

[17] S. Siewert, M. Andalibi, S. Bruder, I. Gentilini, and J. Buchholz, "Drone net architecture for uas traffic management multi-modal sensor networking experiments," in 2018 IEEE Aerospace Conference, 2018, pp. $1-18$.

[18] A. Das, S. Shirazipourazad, D. Hay, and A. Sen, "Tracking of multiple targets using optimal number of uavs," IEEE Transactions on Aerospace and Electronic Systems, vol. 55, no. 4, pp. 1769-1784, 2019.
[19] T. Vidal, G. Laporte, and P. Matl, "A concise guide to existing and emerging vehicle routing problem variants," European Journal of Operational Research, 2019.

[20] G. Laporte, "Fifty years of vehicle routing," Transportation Science, vol. 43, no. 4, p. 408-416, Nov. 2009. [Online]. Available: https://doi.org/10.1287/trsc.1090.0301

[21] B. Eksioglu, A. V. Vural, and A. Reisman, "The vehicle routing problem: A taxonomic review," Computers \& Industrial Engineering, vol. 57, no. 4, pp. 1472-1483, 2009.

[22] J. Caceres-Cruz, P. Arias, D. Guimarans, D. Riera, and A. A. Juan, "Rich vehicle routing problem: Survey," ACM Comput. Surv., vol. 47, no. 2, Dec. 2014.

[23] IBM, IBM ILOG CPLEX Optimization Studio CPLEX User's Manual, Armonk, New York, U.S., 2011.

[24] H. N. Psaraftis, M. Wen, and C. A. Kontovas, "Dynamic vehicle routing problems: Three decades and counting," Networks, vol. 67, no. 1, pp. 3-31, 2016.

[25] A. Richards and J. P. How, "Robust variable horizon model predictive control for vehicle maneuvering," International Journal of Robust and Nonlinear Control, vol. 16, no. 7, pp. 333-351, 2006.

[26] R. J. Afonso, M. R. Maximo, and R. K. Galvão, "Task allocation and trajectory planning for multiple agents in the presence of obstacle and connectivity constraints with mixed-integer linear programming," International Journal of Robust and Nonlinear Control, vol. 30, no. 14, pp. 5464-5491, 2020. [Online]. Available: https://onlinelibrary.wiley.com/doi/abs/10.1002/rnc.5092

[27] R. Siegwart, I. R. Nourbakhsh, and D. Scaramuzza, Introduction to Autonomous Mobile Robots. Cambridge, Massachusetts, USA: The MIT Press, February 2011.

[28] S. M. LaValle, Planning Algorithms. Cambridge, U.K.: Cambridge University Press, 2006, available at http://planning.cs.uiuc.edu/. 